\title{
IncRNAs and circRNAs provide insight into discoid lupus pathogenesis and progression
}

\author{
Michelle Le", Anastasiya Muntyanu", Elena Netchiporouk \\ Division of Dermatology, Department of Medicine, McGill University Health Centre, Montreal, Quebec, Canada \\ \#These authors contributed equally to this work. \\ Correspondence to: Elena Netchiporouk, MD. McGill University Health Centre, 1650 Cedar Ave., Montreal, Quebec H3G 1A4, Canada. \\ Email: elena.netchiporouk@mail.mcgill.ca. \\ Provenance and Peer Review: This article was commissioned by the Editorial Office, Annals of Translational Medicine. The article did not undergo \\ external peer review. \\ Comment on: Xuan J, Xiong Y, Shi L, et al. Do lncRNAs and circRNAs expression profiles influence discoid lupus erythematosus progression?-a \\ comprehensive analysis. Ann Transl Med 2019;7:728.
}

Submitted Feb 09, 2020. Accepted for publication Feb 24, 2020.

doi: $10.21037 / \mathrm{atm} .2020 .03 .56$

View this article at: http://dx.doi.org/10.21037/atm.2020.03.56

Autoimmune diseases are a group of heterogeneous conditions, which develop following the loss of immune tolerance to self-antigens (1). While the underlying molecular mechanisms of these illnesses are not well known, increasing evidence suggests that the complex interplay of environmental factors and epigenetic dysregulation facilitate the pathogenesis in genetically susceptible individuals $(2,3)$.

With the use of next generation sequencing, it has been found that only $\sim 1 \%$ of the human genome is actually transcribed into protein coding messenger RNAs (mRNAs) while most of the genome is transcribed into non-coding RNAs (ncRNAs) (4). In genome-wide association studies, these ncRNAs account for up to $80 \%$ of phenotype-related loci (4).

The long non-coding RNAs (lncRNAs), a type of ncRNAs, are characterized by a length of more than 200 nucleotides and are categorized based on their location relative to the protein-coding genes (4). They are usually located in the intergenic regions with no-protein coding ability, but can occasionally overlap with the protein coding genes (4). Due to their proximity to mRNAs, the expression of lncRNA sequences may be closely correlated with mRNA expression. For instance, it is thought that lncRNAs could silence and/or activate the protein-coding genes thereby impacting their translation (4). Although thousands of IncRNAs are known, only a few have been assigned a corresponding biologic function such as regulation of cell proliferation, apoptosis, and response to stress. Notably, dysregulated expression of lncRNAs has been demonstrated in several diseases including autoimmune thyroid disease and rheumatoid arthritis (RA) (5).

Circular RNAs (circRNAs) are also ncRNAs that can be found anywhere in the genome. They have been implicated in the regulation of many cellular processes including deoxyribonucleic acid (DNA) methylation and the inflammatory response. As such, circRNAs may be involved in the pathogenesis of a number of autoimmune diseases, such as RA, systemic lupus erythematosus (SLE), multiple sclerosis (MS), primary biliary cholangitis (PBC), and have been proposed as potential non-invasive biomarkers for these diseases (6).

Previous studies have shown that $\operatorname{lncRNAs}$ and circRNAs could be implicated in disease development and maintenance in SLE (7-9). Discoid lupus erythematosus (DLE) is a closely related chronic inflammatory disease of the skin which, if left untreated, can lead to significant scarring, disfigurement, and reduced quality of life. DLE appears in about $6-10 \%$ of patients diagnosed with SLE and a similar proportion of DLE patients are at risk of progression to SLE $(10,11)$. It is the most common form of cutaneous lupus accounting for $80 \%$ of cases $(11,12)$. Early diagnosis of the disease and effective treatments could prevent cutaneous and systemic complications and improve the prognosis. 
Xuan et al. are the first to explore the role of lncRNAs and circRNAs in DLE. In their study, they measured the differential expression of mRNA, lncRNA, and circRNA through high-throughput RNA-sequencing (RNA-seq). Three patients with DLE were recruited where a punch biopsy was obtained from each patient's lesional and nonlesional lower lip. Kyoto Encyclopedia of Genes and Genomes (KEGG) and Gene Ontology (GO) pathways analysis, functional coding-noncoding co-expression (CNC) networks, and transcription factor (TF)-lncRNA-enriched mRNA networks were analyzed.

A total of 507 lncRNAs and 62 circRNAs were found to be differentially expressed between affected and normal mucosa. The differential expression of lncRNAs was seen on every chromosome, suggesting that each chromosome contains some abnormalities associated with DLE progression. As demonstrated by the clustering analysis, lncRNA and circRNA expression pattern was different between DLE and matched control tissue. Authors showed a significant correlation between lncRNA and their potential near-by genes by constructing a CNC network using 37 significantly expressed coding genes. Expression of chemokines IL19, CXCL1, CXCL11, and TNFSF15 positively correlated with the up-regulation of lnc-MIPOL1-6, and negatively correlated with the downregulation of lnc-DDX47-3. These chemokines were found to be overexpressed in several autoinflammatory diseases (13-15). In particular, IL19, CXCL11 and TNSF15, have each been shown to be associated with Th1 dominant diseases (13), promote Th1 cell recruitment (16) and stimulate Th1 cytokine production $(2,17)$, respectively. Interestingly, this is the first study to hypothesize that lnc-DDX47-3 and lnc-MIPOL1-6 may be capable of modulating the expression of chemokines important in Th1 signaling and further studies assessing this lncRNA are required to determine their role in disease development.

Given that the function of most lncRNAs has not yet been elucidated, in this analysis, their function was predicted based on the co-expression of mRNAs, which showed that the most significantly upregulated pathways in the DLE samples compared to controls were immune response, inflammatory response, $\mathrm{T}$ cell co-stimulation and chemokine-mediated signaling pathways. Overall, the most upregulated lncRNA in DLE biopsies (>200-fold increase) was HLA complex P5 gene (HCP5). HCP5, an endogenous retrovirus gene, is localized to the major histocompatibility complex (MHC) class I region. It has been found to confer protection against the human immunodeficiency virus (HIV) infection and to increase the risk of autoimmune diseases such as SLE by impairing the immune response and acting as a sponge for miRNAs (18).

Interestingly, the most upregulated protein coding genes were in the staphylococcus aureus infection and viral myocarditis signaling pathways, suggesting a possible infectious implication in disease development and warrant future confirmation. In fact, a previously published case report further supports an association between a reaction to staphylococcus aureus and DLE (19). A study recently published in SLE showed a dysbiosis in SLE skin microbiome favoring staphylococcus aureus (20). No similar studies were conducted so far in DLE. Additionally, viruses were found to be the most frequent pathogens associated with SLE, which is a closely related clinical entity to DLE. Notably, human endogenous retroviruses, Epstein-Barr virus, parvovirus B19, cytomegalovirus and HIV type 1 were demonstrated to trigger the activation of the immune system in SLE (21). Macrophage migration inhibitory factor (MIF), is also an important factor expressed in acute and chronic immuno-inflammatory conditions such as RA, atherosclerosis, and SLE. MIF was found to be upregulated by 13 -fold in lesional DLE skin, which is in keeping with previous studies and could explain some of the inflammatory mechanism behind DLE development (22).

To predict cis and trans regulatory interactions of lncRNA and mRNA expression and TF function, authors took 8 significantly overexpressed TFs and their associated IncRNAs to construct a co-expression network. Each lncRNA connected with 1-10 TFs and each pair of lncRNA-TF affected the expression of several genes. Results show that most of the differentially expressed lncRNAs participated in pathways regulated by TFs, STAT4, ETV6, and ZNF597, suggesting that these TFs are important in the pathogenesis of DLE. STAT4, a cytokine-responsive $\mathrm{TF}$, mediates responses to IL12 and plays a critical role in the differentiation of Th1 cells (23). ETV6, an important TF for blood cell development, has been found to be associated with multiple hematologic malignancies such as primarily acute lymphoblastic lymphoma (24). The function of TF ZNF597, has not yet been elucidated (25). These findings suggest that IncRNA may have transregulatory function and predispose to DLE by altering the transcription of various protein-coding genes. Regarding circRNAs in DLE samples, the strongest signal found in this study implicated protein ubiquitination and TF activity.

To the best of our knowledge, this is the first study investigating the potential role of lncRNA and circRNA 
in the pathogenesis of DLE and contributes to the limited knowledge surrounding the role of ncRNA in autoimmune diseases. An advantage of this study was that affected, and control samples were taken from the same individual hence eliminating heterogeneity in baseline gene expression leading to meaningful results. Similarly, a differential expression of ncRNAs was demonstrated in SLE. For instance, a recent study done by Wu et al., 2019 demonstrated elevated plasma levels of lnc0597, lnc0640, and Inc5150 in SLE patients compared to those of healthy controls (9). Another study by Wang et al., 2019 revealed that $\operatorname{lnc} 7514$ was correlated with SLE disease activity (8) and interaction pairs of IncRNA and nearby targeted mRNA in SLE, NRIR-RSAD2, RP11-153M7.5-TLR2, RP4-758J18.2-CCNL2, RP11-69E11.4-PABPC4 and RP11-496I9.1-IRF7/HRAS/PHRF1 were identified by Cao et al., 2019 (7). Nevertheless, studies investigating the role of ncRNA in SLE are limited and future studies may demonstrate an overlap between ncRNA profiles of SLE and DLE given their close clinical relation.

Although this avant-garde study shows potential both in basic science research and in clinical application, its limitations include the small number of participants included. As such, a global diversity of participants is excluded and interpreted results can only be applied to select individuals. Future studies with a larger sample size investigating the differential expression of lncRNA and circRNA in patients with DLE are warranted. With larger studies, clinical application of these results could include the role of lncRNAs and circRNAs as biomarkers for disease development, targets for genetic testing for the diagnosis of DLE, or for following the progression of the disease in patients with DLE. Molecular targeting using knockout lnRNA/circRNA in in vivo animal models or in vitro cell lines may be beneficial in discovering the role of critical lncRNAs and circRNAs and assessing their involvement in disease development. These studies would increase our understanding of the molecular mechanisms that underlie autoimmune diseases and may be used to define critical lncRNAs and circRNAs, which could be used as future drug targets for DLE. Furthermore, given the strong familial history among autoimmune diseases, it would be of interest to investigate if these genetic expression profiles suggest a familial inheritance pattern.

Overall, the significance and role of the differential expression of lncRNAs and circRNAs in DLE and other autoimmune diseases is in its infancy and calls for future research.

\section{Acknowledgments}

Funding: None.

\section{Footnote}

Conflicts of Interest: The authors have no conflicts of interest to declare.

Ethical Statement: The authors are accountable for all aspects of the work in ensuring that questions related to the accuracy or integrity of any part of the work are appropriately investigated and resolved.

Open Access Statement: This is an Open Access article distributed in accordance with the Creative Commons Attribution-NonCommercial-NoDerivs 4.0 International License (CC BY-NC-ND 4.0), which permits the noncommercial replication and distribution of the article with the strict proviso that no changes or edits are made and the original work is properly cited (including links to both the formal publication through the relevant DOI and the license). See: https://creativecommons.org/licenses/by-nc-nd/4.0/.

\section{References}

1. Sharif K, Amital H, Shoenfeld Y. The role of dietary sodium in autoimmune diseases: The salty truth. Autoimmun Rev 2018;17:1069-73.

2. Zhang Z, Zhang R. Epigenetics in autoimmune diseases: Pathogenesis and prospects for therapy. Autoimmun Rev 2015;14:854-63.

3. Watad A, Azrielant S, Bragazzi NL, et al. Seasonality and autoimmune diseases: The contribution of the four seasons to the mosaic of autoimmunity. J Autoimmun 2017;82:13-30.

4. Li LJ, Zhao W, Tao SS, et al. Comprehensive long noncoding RNA expression profiling reveals their potential roles in systemic lupus erythematosus. Cell Immunol 2017;319:17-27.

5. Atianand MK, Caffrey DR, Fitzgerald KA. Immunobiology of Long Noncoding RNAs. Annu Rev Immunol 2017;35:177-98.

6. Zhou Z, Sun B, Huang S, et al. Roles of circular RNAs in immune regulation and autoimmune diseases. Cell Death Dis 2019;10:503.

7. Cao H, Li D, Lu H, et al. Uncovering potential lncRNAs and nearby mRNAs in systemic lupus erythematosus from the Gene Expression Omnibus dataset. Epigenomics 
2019;11:1795-809.

8. Wang JB, Li J, Zhang TP, et al. Expression of several long noncoding RNAs in peripheral blood mononuclear cells of patients with systemic lupus erythematosus. Adv Med Sci 2019;64:430-6.

9. Wu GC, Hu Y, Guan SY, et al. Differential Plasma Expression Profiles of Long Non-Coding RNAs Reveal Potential Biomarkers for Systemic Lupus Erythematosus. Biomolecules 2019. doi: 10.3390/biom9060206.

10. Mendez-Flores S, Hernandez-Molina G, Azamar-Llamas $\mathrm{D}$, et al. Inflammatory chemokine profiles and their correlations with effector CD4 $\mathrm{T}$ cell and regulatory cell subpopulations in cutaneous lupus erythematosus. Cytokine 2019;119:95-112.

11. Sole C, Domingo S, Ferrer B, et al. MicroRNA Expression Profiling Identifies miR-31 and miR-485-3p as Regulators in the Pathogenesis of Discoid Cutaneous Lupus. J Invest Dermatol 2019;139:51-61.

12. Ghauri AJ, Valenzuela AA, O'Donnell B, et al. Periorbital discoid lupus erythematosus. Ophthalmology 2012;119:2193-4.e11.

13. Azuma YT, Nakajima H, Takeuchi T. IL-19 as a potential therapeutic in autoimmune and inflammatory diseases. Curr Pharm Des 2011;17:3776-80.

14. Rotondi M, Chiovato L, Romagnani S, et al. Role of chemokines in endocrine autoimmune diseases. Endocr Rev 2007;28:492-520.

15. Aiba Y, Nakamura M. The role of TL1A and DR3 in autoimmune and inflammatory diseases. Mediators Inflamm 2013;2013:258164.

16. Kumar RK, Herbert C. Chapter 11 - Airway Epithelial Cytokines in Asthma and Chronic Obstructive Pulmonary Disease. In: Foti M, Locati M, editors. Cytokine Effector

Cite this article as: Le M, Muntyanu A, Netchiporouk E. IncRNAs and circRNAs provide insight into discoid lupus pathogenesis and progression. Ann Transl Med 2020;8(6):260. doi: 10.21037/atm.2020.03.56
Functions in Tissues. Cambridge, Massachusetts: Academic Press, 2017:163-72.

17. Zhang Z, Li LY. TNFSF15 Modulates Neovascularization and Inflammation. Cancer Microenviron 2012;5:237-47.

18. Chen R, Xin G, Zhang X. Long non-coding RNA HCP5 serves as a ceRNA sponging miR-17-5p and miR-27a/b to regulate the pathogenesis of childhood obesity via the MAPK signaling pathway. J Pediatr Endocrinol Metab 2019;32:1327-39.

19. Grice K, Doniach D, Turk JL. Discoid lupus erythematosus at the site of an intradermal injection of killed Staphylococcus aureus. Clin Exp Immunol 1967;2:489-95.

20. Huang C, Yi X, Long H, et al. Disordered cutaneous microbiota in systemic lupus erythematosus. J Autoimmun 2020;108:102391.

21. Illescas-Montes R, Corona-Castro CC, MelguizoRodríguez L, et al. Infectious processes and systemic lupus erythematosus. Immunology 2019;158:153-60.

22. Santos LL, Morand EF. Macrophage migration inhibitory factor: a key cytokine in RA, SLE and atherosclerosis. Clin Chim Acta 2009;399:1-7.

23. Jordan MA, Field J, Butzkueven H, et al. Chapter 26 Genetic Predisposition, Humans. In: Rose NR, Mackay IR, editors. The Autoimmune Diseases. Fifth edition. Boston: Academic Press, 2014:341-64.

24. Lambert MP. Chapter 93 - Congenital Thrombocytopenia. In: Shaz BH, Hillyer CD, Reyes Gil M, editors. Transfusion Medicine and Hemostasis. Third edition. Amsterdam: Elsevier; 2019:571-80.

25. Schulze KV, Szafranski P, Lesmana H, et al. Novel parent-of-origin-specific differentially methylated loci on chromosome 16. Clin Epigenetics 2019;11:60. 